\title{
Sazonalidade e potencial energético da biomassa residual agrícola na região dos Campos Gerais do Paraná
}

\author{
Letícia de Pierri ${ }^{1^{*}}$, Volnei Pauletti ${ }^{2 *}$, Dimas Agostinho da Silva ${ }^{3}$, Clewerson Frederico Scheraiber ${ }^{4}$, \\ Jorge Luiz Moretti de Souza ${ }^{5}$,Fernando Cesar Munaro ${ }^{6}$
}

$10.1590 / 0034-737 X 201663020003$

\section{RESUMO}

Teve-se por objetivo, neste trabalho, avaliar as disponibilidades sazonal e espacial da biomassa residual da colheita das principais culturas de grãos, na região dos Campos Gerais, PR, estimar o potencial teórico de produção de energia elétrica a partir desses resíduos, bem como identificar os municípios da região com maior potencial para instalação de termelétricas movidas a biomassa. Dados de produtividade de milho, soja, trigo, cevada, aveia branca e aveia preta foram obtidos, na Secretaria de Agricultura do Paraná, para as safras 2008/2009 e 2009/2010 e, a partir do índice e do calendário de colheitas, estimou-se a produção e sazonalidade da biomassa residual das culturas analisadas. Foram coletadas amostras de biomassa destas espécies e determinou-se o poder calorífico superior $(P C S)$ e o potencial teórico de produção de energia elétrica a partir dos resíduos quantificados. Os Campos Gerais do Paraná produziram 3.862.130 (2008/2009) e 4.107.932 Mg ha"1 (2009/2010) de biomassa residual total com o cultivo das seis culturas. A disponibilidade de biomassa concentra-se em março e abril (aproximadamente 60\%), sendo inexpressiva em dezembro, janeiro e entre junho e agosto. A biomassa residual da colheita de grãos apresentou PCS comparável ao de espécies florestais e potencial total de produção de energia de 3.932 (2008/2009) e 4.185 GWh ano"1 (2009/2010). Dos municípios analisados, Tibagi e Castro são os mais promissores para a instalação de termelétricas movidas a biomassa, por apresentarem maior produção e melhor distribuição sazonal dos resíduos.

Palavras-chave: palha, bioenergia, resíduo da agricultura.

\section{ABSTRACT \\ Seasonality and potential of energy production from agricultural residual biomass in Campos Gerais, Paraná State}

This study aimed to evaluate the seasonal and spatial availability of harvesting residual biomass of the main grain crops in the region of Campos Gerais - PR, estimate the theoretical potential to electricity production from these residues, as well as identify cities in the region with greater potential for installation of biomass-fueled thermoelectric . Yield data of corn, soybean, wheat, barley, oat and black oat were obtained from the Secretary of Agriculture of Paraná, for the seasons 2008/2009 and 2009/2010, and through the index and harvest calendar, the production and seasonality of total residual biomass of these crops were estimated. Biomass samples were collected from these species and their higher heating value (HHV) and theoretical potential to electricity production were determined. Campos Gerais produced 3.862.130 (2008/2009) and 4.107.932 $\mathrm{Mg} \mathrm{ha}^{-1}$ (2009/2010) of total residual biomass with the six crops evaluated. The

\footnotetext{
Submetido em 27/11/2014 e aprovado em 04/01/2016

' Universidade de São Paulo, Campus "Luiz de Queiroz", Departamento de Ciência do Solo, Piracicaba, São Paulo, Brasil. leticiapierri@usp.br

${ }^{2}$ Universidade Federal do Paraná, Departamento de Solos e Engenharia Agrícola, Curitiba, Paraná, Brasil. vpauletti@ufpr.br

${ }^{3}$ Universidade Federal do Paraná, Departamento de Engenharia Florestal, Curitiba, Paraná, Brasil. dimas.agostinho.silva@gmail.com

${ }^{4}$ Universidade Federal do Paraná, Departamento de Solos e Engenharia Agrícola, Curitiba, Paraná, Brasil.clewerson.fred@gmail.com

${ }^{5}$ Universidade Federal do Paraná, Departamento de Solos e Engenharia Agrícola, Curitiba, Paraná, Brasil. jmoretti@ufpr.br

${ }^{6}$ Universidade Federal do Paraná, Departamento de Solos e Engenharia Agrícola, Curitiba, Paraná, Brasil. fernandocmunaro@ gmail.com

*Autora para correspondência: leticiapierri@usp.br
} 
availability of biomass was concentrated in March and April (about 60\%), being negligible in December, January and between June and August. The residual biomass of harvesting grain showed an HHV comparable to forest species, with a total potential energy production of 3.932 (2008/2009) and 4.185 GWh year-1 (2009/2010). Among the cities, Tibagi and Castro are the most promising for the installation of a biomass-fueled thermoelectric, due to their larger production and better seasonal distribution of residuals.

\section{INTRODUÇÃO}

Diante da crescente busca por fontes de energia renovável, a biomassa tem-se mostrado particularmente promissora, no Brasil, o que resultou em aumento de $14,5 \%$ da participação dessa fonte na matriz elétrica nacional, de 2012 para 2013 (EPE, 2014). Em relação a outros países, o Brasil apresenta alto potencial de exploração da biomassa residual da colheita de culturas anuais para produção de energia, graças ao grande volume de produção de biomassa produzida (Lora \& Andrade, 2009; Valdez-Vasquez et al., 2010; Okello et al., 2013), o que se torna importante, principalmente nos períodos de baixa vazão das hidrelétricas.

O potencial da biomassa residual agrícola, no entanto, ainda é pouco explorado por causa da preocupação existente em relação aos possíveis efeitos negativos sobre a conservação do solo, advindos de sua retirada (Tarkalson et al., 2009; Kludze et al., 2013; Leal et al., 2013), além dos impactos em relação a importantes aspectos do solo, como a ciclagem de nutrientes, a atividade microbiana, o controle de plantas daninhas, a estabilidade de agregados, dentre outros. Por outro lado, trabalhos têm mostrado que a destinação de biomassa residual agrícola para produção de energia é ambientalmente segura e que a remoção parcial de 25 a $30 \%$ do total produzido tem pouco efeito sobre as perdas de solo por erosão (Powers et al., 2011; Leal et al., 2013) e de matéria orgânica (Andrews, 2006). Regiões que apresentam produtividade de grãos acima da média agrícola nacional, como os Campos Gerais do Paraná (Shimandeiro et al., 2008), destacam-se, neste sentido, pois podem aproveitar o material disponível e já produzido para atender demandas locais de energia elétrica, sem comprometer a produção de alimentos e a qualidade do solo (Okello et al., 2013).

Diversos levantamentos do potencial de produção de energia a partir da biomassa têm sido realizados no mundo. Parikka (2004) quantificou a biomassa global disponível para fins energéticos, mas limitou-se apenas aos resíduos madeireiros, desconsiderando o expressivo potencial existente nos resíduos agrícolas, que incluem resíduos da colheita como palhas, colmos, cascas, talos, dentre outros. Para esses tipos de biomassa, levantamentos regionais foram realizados em países que apresentam alta disponibilidade de resíduos, como China (Cuiping et al., 2004),
Japão (Matsumura et al., 2005), Romênia (Scarlat et al., 2011), Uganda (Okello et al., 2013) e Austrália (Kingwell \& Abadi, 2014).

No Brasil, um importante trabalho foi desenvolvido por Lora \& Andrade (2009), apresentando as tecnologias de conversão disponíveis e o potencial de vários tipos de biomassa como fonte energética no país. No entanto, como a produtividade de resíduos depende do local de cultivo, relacionado com as variações de clima e solo (Cuiping et al., 2004), pode ter havido subestimativa do potencial em algumas regiões que apresentam alto volume de produção agrícola. A principal vantagem de levantamentos locais é a promoção de informações que suportem decisões estratégicas (Okello et al., 2013), como a escolha de microrregiões ou municípios (Scarlat et al., 2011; Kingwell \& Abadi, 2014) que apresentem logística favorável à instalação de plantas de bioenergia. Assim, um dos passos mais importantes no planejamento de cadeias de suprimento de energia a partir de biomassa residual é a avaliação de sua disponibilidade espacial e temporal (Scarlat $e t$ al., 2011; Okello et al., 2013), provendo informações para o gerenciamento e a exploração dessa fonte energética (Correll et al., 2014; Kingwell \& Abadi, 2014).

Teve-se por objetivo, neste trabalho, avaliar as disponibilidades sazonal e espacial da biomassa dos resíduos oriundos da colheita das principais culturas de grãos na região dos Campos Gerais, PR, estimar o potencial teórico de produção de energia elétrica a partir desses resíduos, bem como identificar os municípios da região com maior potencial para instalação de termelétricas movidas a biomassa.

\section{MATERIAL E MÉTODOS}

Para a delimitação geofísica da região em estudo, consideraram-se os seguintes municípios: Arapoti, Balsa Nova, Campo do Tenente, Campo Largo, Cândido de Abreu, Carambeí, Castro, Imbaú, Ipiranga, Ivaí, Jaguariaíva, Lapa, Ortigueira, Palmeira, Piraí do Sul, Ponta Grossa, Porto Amazonas, Reserva, Rio Negro, São José da Boa Vista, Telêmaco Borba, Teixeira Soares, Tibagi e Ventania (UEPG, 2011). Segundo a classificação de Köeppen, os Campos Gerais do Paraná apresentam clima variando entre subtropical, com verões quentes e chuvas concentradas no verão (Cfa), e temperado, com verões frescos sem estação seca defini- 
da (Cfb). As duas classes de solos predominantes na região são Cambissolos e Latossolos (Sá et al., 2012). Apesar da grande variação topográfica, a atividade agrícola desenvolve-se em mais de $5.000 \mathrm{~km}^{2}$, geralmente em terrenos com relevo suave ondulado (Melo et al., 2007). Dados históricos de produtividade do Instituto Brasileiro de Geografia e Estatística (IBGE) indicam que a região dos Campos Gerais Paranaenses destaca-se na produção das culturas de milho (Zea mays L.) e soja (Glycine max L. Merr.), na safra de verão, e das culturas de trigo (Triticum aestivum L.), cevada (Hordeum vulgare L.), aveia branca (Avena sativa L.) e aveia preta (Avena strigosa Schreb.), na safra de inverno (IBGE, 2011). Desta maneira, foram analisados os dados de produção total de grãos, de área colhida e de produtividade de grãos dessas espécies agrícolas, para cada município da região, nas safras de 2008/2009 e 2009/ 2010. Os dados são provenientes da Secretaria de Estado de Agricultura e Abastecimento (SEAB/DERAL - Departamento de Economia Rural) (SEAB, 2011a).

Após a obtenção dos dados individuais de cada município, calculou-se o total de produção de grãos da região, fazendo-se a soma de todos os valores. Posteriormente, realizou-se o cálculo da produção de biomassa residual, levando-se em consideração o índice de colheita (IC) de cada cultura, que representa a quantidade de grãos presentes na biomassa aérea total da planta. $\mathrm{O}$ cálculo da produção de biomassa residual é apresentado na Equação (1):

$$
P B R=\frac{P G}{I C}-P G
$$

sendo: $P B R$ - produtividade de biomassa residual $\left(\mathrm{kg} \mathrm{ha}^{-1}\right)$; $P G$ - produtividade de grãos $\left(\mathrm{kg} \mathrm{ha}^{-1}\right), I C$ - índice de colheita.

Foram calculados, também, a área colhida total e a produtividade média de grãos de cada cultura da região, por meio da soma e médias dos dados de cada um dos 24 municípios. Para a análise da sazonalidade de biomassa residual, considerou-se o valor total de produção de biomassa dos municípios e o calendário de colheita das culturas agrícolas do Paraná (SEAB, 2011b). Os dados foram dispostos na forma de percentagem acumulada de área colhida, sendo utilizados para a determinação da quantidade de biomassa residual disponível, em cada mês do ano, para as duas safras (Tabela 1).

A partir dos dados de sazonalidade, elaborou-se um mapa com o software gráfico ArcGis 10 (ESRI, 2008), em que se considerou a produção total de biomassa (soma de todas as culturas) para cada município analisado, sendo os dados agrupados em trimestres e apresentados em percentagem do total produzido.

Para determinar o potencial teórico de produção de eletricidade, a partir dos resíduos quantificados, amostras de biomassa residual provenientes da região dos Campos
Gerais e de municípios próximos foram coletadas, sendo: Itaberá-SP (milho), Arapoti-PR e Ponta Grossa-PR (soja) e Santa Izabel do Oeste-PR (trigo, aveia branca e aveia preta). Por causa da semelhança botânica e da similaridade em relação ao poder calorífico (Dodiæet al., 2011; Carroll \& Finnan, 2012), consideraram-se as amostras de trigo equivalentes às de cevada. As amostras foram secadas em estufa de ventilação forçada, a $60{ }^{\circ} \mathrm{C}$, moídas em moinho de facas modelo Willey e novamente secadas em estufa, a 103 ${ }^{\circ} \mathrm{C}$ por $24 \mathrm{~h}$. Procedeu-se à análise do PCS em um calorímetro adiabático modelo IKA-WERKE® C5000 (ABNT, 1984). A conversão de energia térmica $(P C S)$, em $\mathrm{kJ} \mathrm{kg}^{-1}$, para unidade de energia elétrica $(\mathrm{kWh})$, foi realizada, considerando-se que $3.600 \mathrm{~kJ}$ equivalem a $1 \mathrm{kWh}$ (EPE, 2011). Posteriormente, para o cálculo do potencial teórico de produção de energia, considerou-se a eficiência média de caldeiras de $20 \%$ (Nogueira \& Lora, 2003).

\section{RESULTADOS E DISCUSSÃO}

\section{Produção de biomassa residual}

As produtividades de grãos alcançadas nos Campos Gerais ficaram acima da média nacional para a mesma safra, em relação às culturas do milho, soja, cevada e trigo $(\mathrm{CONAB}$, 2013) (Tabela 2). Apesar de representar apenas 5,9\% da área do Estado do Paraná, a região dos Campos Gerais mostrou significativa produção de biomassa agrícola, tendo em vista que o índice de colheita das culturas variou em torno de $50 \%$. Os resíduos da produção agrícola são dependentes do tipo e da variedade da cultura semeada e de suas produtividades (Karaj et al., 2010), sendo reflexo do local de cultivo, determinado pelas variações climáticas e de solo (Cuiping et al., 2004; Fowler et al., 2009). Os resultados obtidos (Tabela 2) indicam que a região dos Campos Gerais apresenta condições favoráveis ao bom desenvolvimento agrícola e à produção e exploração energética da biomassa de culturas anuais frente à média nacional.

A maior contribuição da produção total de biomassa está associada ao milho e à soja, contribuindo com aproximadamente $80 \%$ de toda biomassa produzida pelas espécies avaliadas, considerando-se as duas safras (Tabela 2). A soja foi a espécie mais cultivada na região, enquanto o milho, apesar de menor área de cultivo, apresenta alta produtividade de biomassa, sendo a espécie com maior potencial de contribuição para geração de energia a partir de sua biomassa residual.

Entre os cultivos de inverno, o trigo foi o principal produtor de biomassa e a cevada, pela menor área, a que menos contribuiu (Tabela 2). Por outro lado, a aveia preta, apesar de menor produtividade média em relação à do trigo, apresentou área de cultivo expressiva, o que contribuiu significativamente para o total de biomassa produzida. A menor contribuição individual das espécies de inver- 
no era esperada, visto que apresentam área menor colhida, em relação ao milho e à soja. Desta forma, o potencial de exploração de biomassa para fins energéticos, entre as culturas avaliadas, diminuiu na seguinte sequência: milho $>$ soja > trigo > aveia preta > aveia branca > cevada.

A aveia preta é uma espécie utilizada geralmente para produção de biomassa para cobertura do solo e ciclagem de nutrientes, especialmente no sistema plantio direto, ou mesmo para pastejo por animais (Scarlat et al., 2011). Como a área total cultivada para produção de grãos no inverno é aproximadamente um terço da área cultivada no verão (Tabela 2) e as variedades atuais podem apresentar produtividades superiores a $5.000 \mathrm{~kg}$ ha"1 de biomassa seca (Noro et al., 2003), acredita-se que a aveia preta pode ser uma espécie interessante, servindo ao propósito agronômico na rotação de culturas e gerando biomassa para ser utilizada na produção de energia, no inverno, sem concorrer por área para a produção de grãos.

Tabela 1: Percentagem acumulada de área colhida, ao longo do ano, das culturas de grãos do Estado do Paraná, Brasil, nas safras 2008/ 2009 e 2009/2010

\begin{tabular}{|c|c|c|c|c|c|c|c|c|c|c|c|c|}
\hline \multirow{2}{*}{ Cultura } & \multicolumn{12}{|c|}{ Área colhida (\%) } \\
\hline & Jan & Fev & Mar & Abr & Mai & Jun & Jul & Ago & Set & Out & Nov & Dez \\
\hline \multicolumn{13}{|c|}{$2008 / 2009$} \\
\hline Milho & 1 & 25 & 56 & 85 & 98 & 99 & 100 & - & - & - & - & - \\
\hline Soja & - & 1 & 51 & 92 & 100 & - & - & - & - & - & - & - \\
\hline Trigo & - & - & - & - & - & - & - & 1 & 32 & 78 & 97 & 100 \\
\hline Cevada & - & - & - & - & - & - & 一 & - & 1 & 17 & 92 & 100 \\
\hline Aveia Branca & - & - & - & - & - & - & - & 5 & 54 & 85 & 99 & 100 \\
\hline Aveia Preta & - & - & - & - & - & - & - & 2 & 38 & 83 & 98 & 100 \\
\hline \multicolumn{13}{|c|}{$2009 / 2010$} \\
\hline Milho & 2 & 23 & 61 & 92 & 97 & 99 & 100 & - & - & - & - & - \\
\hline Soja & 1 & 17 & 75 & 98 & 100 & - & - & - & - & - & - & - \\
\hline Trigo & - & - & - & - & - & - & - & 9 & 67 & 85 & 97 & 100 \\
\hline Cevada & - & - & - & - & - & - & - & - & 3 & 23 & 95 & 100 \\
\hline Aveia Branca & - & - & - & - & - & - & 1 & 10 & 75 & 89 & 99 & 100 \\
\hline Aveia Preta & - & - & - & - & - & - & - & 7 & 65 & 91 & 99 & 100 \\
\hline
\end{tabular}

Fonte: Secretaria da Agricultura e do Abastecimento do Paraná (SEAB, 2011b).

Tabela 2: Produção total de grãos (PTG), área colhida (AC), produtividade média de grãos (PG), índice de colheita (IC) e produção total de biomassa (PTB) das principais culturas cultivadas nos 24 municípios da Região dos Campos Gerais, PR, Brasil, nas safras 2008/2009 e 2009/2010

\begin{tabular}{|c|c|c|c|c|c|c|}
\hline \multirow{2}{*}{ Espécie } & \multirow{2}{*}{$\begin{array}{l}P T G \\
(\mathrm{Mg})\end{array}$} & \multirow{2}{*}{$\begin{array}{l}A C \\
\text { (ha) }\end{array}$} & \multirow{2}{*}{$\begin{array}{c}P G \\
\text { (kg ha"1) }\end{array}$} & \multirow{2}{*}{$\begin{array}{c}I C^{(1)} \\
\text { (adimensional) }\end{array}$} & \multicolumn{2}{|c|}{$P T B$} \\
\hline & & & & & (Mg) & $(\%)$ \\
\hline \multicolumn{7}{|c|}{$2008 / 2009$} \\
\hline Milho & 1.663 .817 & 250.185 & 6.519 & 0,49 & 1.731 .728 & 44,8 \\
\hline Soja & 1.456 .321 & 502.510 & 2.929 & 0,52 & 1.344 .296 & 34,8 \\
\hline Trigo & 507.624 & 163.360 & 2.815 & 0,53 & 450.157 & 11,7 \\
\hline Cevada & 45.751 & 13.542 & 2.996 & 0,61 & 29.250 & 0,8 \\
\hline Aveia Branca & 64.100 & 21.215 & 2.714 & 0,56 & 50.364 & 1,3 \\
\hline Aveia Preta & 85.445 & 52.900 & 1.578 & 0,25 & 256.335 & 6,6 \\
\hline Total & 3.823 .058 & 1.003 .712 & - & - & 3.862 .130 & 100 \\
\hline \multicolumn{7}{|c|}{$2009 / 2010$} \\
\hline Milho & 1.673 .149 & 192.620 & 8.407 & 0,49 & 1.741 .440 & 42,4 \\
\hline Soja & 1.724 .362 & 555.030 & 3.046 & 0,52 & 1.591 .718 & 38,7 \\
\hline Trigo & 490.437 & 132.060 & 3.371 & 0,53 & 434.916 & 10,6 \\
\hline Cevada & 57.068 & 15.780 & 3.379 & 0,61 & 36.486 & 0,9 \\
\hline Aveia Branca & 81.572 & 23.800 & 3.000 & 0,56 & 64.092 & 1,6 \\
\hline Aveia Preta & 79.760 & 53.650 & 1.406 & 0,25 & 239.280 & 5,8 \\
\hline Total & 4.106.348 & 972.940 & - & - & 4.107.932 & 100 \\
\hline
\end{tabular}

(1) Informação fornecida por pesquisadores, levantada em propriedades da região dos Campos Gerais. 
A safra do ano agrícola de 2009/2010 apresentou maior produção de biomassa residual do que a safra 2008/2009. A principal causa dessa diferença foi o déficit hídrico ocorrido durante o cultivo de verão, em 2008/2009, com precipitação pluviométrica $62 \%$ menor do que a média histórica para a região. Para a safra 2009/10, durante o mesmo período, a precipitação foi $22 \%$ superior à média histórica (Silvestrin, 2011). Observou-se, também, que parte da área destinada ao cultivo de milho (aproximadamente 58.000 ha) foi utilizada para a soja, no ano seguinte, provavelmente pela perspectiva de preços a serem pagos ao produtor. A variação de clima e de área de cultivo das espécies reflete diretamente na oferta de biomassa e deve ser considerada no planejamento das termelétricas, a longo prazo (Kingwell \& Abadi, 2014).

\section{Sazonalidade da biomassa residual}

A maior disponibilidade de biomassa, nas duas safras avaliadas, ocorreu entre fevereiro e maio, principalmente nos meses de março e abril (Tabela 3), intervalo em que a maior parte das áreas de milho e de soja é colhida (Tabela 1). Outro período de grande oferta de biomassa é durante a colheita das espécies de inverno, nos meses de setembro e outubro. Em termos percentuais, houve concentração de mais da metade da produção de biomassa nos meses de março $(\approx 34 \%)$ e abril $(\approx 24,5 \%)$, enquanto a menor oferta ocorreu no inverno (junho, julho e agosto) e parte do verão (dezembro e janeiro), com média geral de 0,64\% ao mês (Figura 1). Os meses com menor produção de biomassa coincidem com aqueles em que, praticamente, não ocorrem colheita das espécies utilizadas para produção de grãos na região (Tabela 1).

Considerando-se uma distribuição homogênea da biomassa durante o ano $(8,3 \%$, ao mês, da produção total de cada safra), verificou-se excedente médio de oferta de 438.358 Mg, durante o período de maior disponibilidade, e déficit de $260.890 \mathrm{Mg}$, no restante do ano (Figura 1). A concentração na produção e, consequentemente, na oferta de biomassa, evidencia a importância do planejamento da disponibilidade de matéria-prima vegetal utilizada como insumo em caldeiras, a fim de otimizá-la (Evans et al., 2010). Portanto, são necessárias alternativas para contornar a falta de biomassa agrícola, durante alguns meses do ano, como a estocagem in natura, em briquetes ou pellets

Tabela 3: Disponibilidade média de biomassa residual agrícola ao longo do ano, das principais culturas cultivadas nos 24 municípios da região dos Campos Gerais, PR, Brasil, nas safras 2008/2009 e 2009/2010

\begin{tabular}{|c|c|c|c|c|c|c|c|}
\hline \multirow{2}{*}{ Mês } & \multicolumn{6}{|c|}{ Disponibilidade média de biomassa agrícola das culturas (Mg) } & \multirow{2}{*}{$\frac{\text { Total }}{\text { (Mg) }}$} \\
\hline & Milho & Soja & Trigo & Cevada & Aveia branca & Aveia preta & \\
\hline \multicolumn{8}{|c|}{$2008 / 2009$} \\
\hline Janeiro & 17.317 & - & - & - & - & - & 17.317 \\
\hline Fevereiro & 415.615 & 13.443 & - & - & - & - & 429.058 \\
\hline Março & 536.836 & 672.148 & - & - & - & - & 1.208 .984 \\
\hline Abril & 502.201 & 551.161 & - & - & - & - & 1.053 .363 \\
\hline Maio & 225.125 & 107.544 & - & - & - & - & 332.668 \\
\hline Junho & 17.317 & - & - & - & - & - & 17.317 \\
\hline Julho & 17.317 & - & - & - & - & - & 17.317 \\
\hline Agosto & - & - & 4.502 & 0 & 2.518 & 5.127 & 12.146 \\
\hline Setembro & - & - & 139.549 & 293 & 24.678 & 92.281 & 256.800 \\
\hline Outubro & - & - & 207.072 & 4.680 & 15.613 & 115.351 & 342.716 \\
\hline Novembro & - & - & 85.530 & 21.938 & 7.051 & 38.450 & 152.969 \\
\hline Dezembro & - & - & 13.505 & 2.340 & 504 & 5.127 & 21.475 \\
\hline \multicolumn{8}{|c|}{$2009 / 2010$} \\
\hline Janeiro & 34.829 & 15.917 & - & - & - & - & 50.746 \\
\hline Fevereiro & 365.702 & 254.675 & - & - & - & - & 620.377 \\
\hline Março & 661.747 & 923.197 & - & - & - & - & 1.584 .944 \\
\hline Abril & 539.846 & 366.095 & - & - & - & - & 905.942 \\
\hline Maio & 87.072 & 31.834 & - & - & - & - & 118.906 \\
\hline Junho & 34.829 & - & - & - & - & - & 34.829 \\
\hline Julho & 17.414 & - & - & - & 641 & - & 18.055 \\
\hline Agosto & - & - & 39.142 & - & 5.768 & 16.750 & 61.660 \\
\hline Setembro & - & - & 252.251 & 1.095 & 41.660 & 138.782 & 433.788 \\
\hline Outubro & - & - & 78.285 & 7.297 & 8.973 & 62.213 & 156.768 \\
\hline Novembro & - & - & 52.190 & 26.270 & 6.409 & 19.142 & 104.011 \\
\hline Dezembro & - & - & 13.047 & 1.824 & 641 & 2.393 & 17.905 \\
\hline
\end{tabular}


(Höldrich et al., 2009; Dodiæet al., 2011; Correll et al., 2014), ou, ainda, a combustão simultânea da biomassa com matéria vegetal lenhosa (cascas de pinus e de eucalipto, por exemplo), ou carvão, durante a entressafra (Matsumura et al., 2005).

Entre os municípios avaliados, Tibagi e Castro destacaram-se como principais produtores de biomassa residual, além de apresentarem distribuição mais homogênea da biomassa, durante os quatro trimestres do ano (Figura 2). A maior homogeneidade de produção decorre da maior expressividade das espécies de inverno, em relação aos municípios vizinhos. As avaliações indicaram que a operação de termelétricas à base de biomassa agrícola, na região dos Campos Gerais, mostra-se vantajosa nos dois municípios.

Os municípios de Campo Largo, Balsa Nova e Telêmaco Borba, por outro lado, apresentaram produção de biomassa proveniente da produção de grãos, exclusivamente nos seis primeiros meses do ano (Figura 2), o que constituiria uma dificuldade para a exploração dessa biomassa para fins energéticos. O transporte da matéria-prima também se mostra um fator determinante na logística de termelétricas movidas a biomassa (Ruiz et al., 2013; Kingwell \& Abadi, 2014). Como a biomassa agrícola apresenta baixa densidade (Fowler $e t$ al., 2009), considera-se que $5 \mathrm{~km}$ é a distância máxima de percurso entre a fonte de matéria-prima e as caldeiras, para que a atividade não se torne economicamente inviável pelo próprio custo de transporte (Höldrich et al., 2009). Contudo, segundo Ruiz et al. (2013), essa distância pode variar para valores superiores a $25 \mathrm{~km}$, o que depende do tamanho e da capacidade instalada de cada planta de energia.

\section{Potencial teórico de produção de eletricidade}

O poder calorífico superior $(P C S)$ das biomassas avaliadas variou entre 17,3 (trigo e cevada) e 18,6 MJ Kg"1 (milho) (Tabela 4), mostrando-se comparável ao das espécies florestais comumente utilizadas na produção de lenha, como pinus e eucalipto, com valores de $P C S$ de $20,2 \mathrm{e}$ 17,6 MJ Kg"1 , respectivamente (Telmo \& Lousada, 2011). Em decorrência da maior produção de biomassa e por apresentarem PCS mais elevado, as culturas do milho e da soja apresentaram potenciais de produção de energia superiores aos das demais culturas avaliadas, correspondendo a mais de $80 \%$ do potencial total disponível.

O potencial teórico total de produção de energia elétrica, com os resíduos agrícolas das seis culturas estudadas, foi estimado em aproximadamente 3.932 e $4.185 \mathrm{GWh}$, para as safras 2008/2009 e 2009/2010, respectivamente (Tabela 4). O valor estimado de produção de energia elétrica supera a demanda local e poderia suprir aproximadamente $24 \%$ do consumo nacional de eletricidade do setor agropecuário, em 2010, que foi igual a $17.573 \mathrm{GWh}$ (EPE, 2011). Considerando-se que a eficiência de conversão termelétrica pode variar de 20 a 40\% (Evans et al., 2010), a produção líquida de eletricidade, a partir dos resíduos quantificados, poderia ainda ser superior aos valores calculados, se considerada uma termelétrica de alta eficiência energética. Contudo, é importante destacar que a estimativa não considerou a umidade dos resíduos, além do fato de que se trata do potencial total, ou seja, de toda a biomassa produzida pelas seis culturas. Como o plantio direto predomina nos Campos Gerais (Briedis et al., 2012), grande parte da biomassa residual da colheita precisa ser mantida no solo para a manutenção do sistema e, portanto, o potencial real de exploração desta biomassa pode ser inferior.

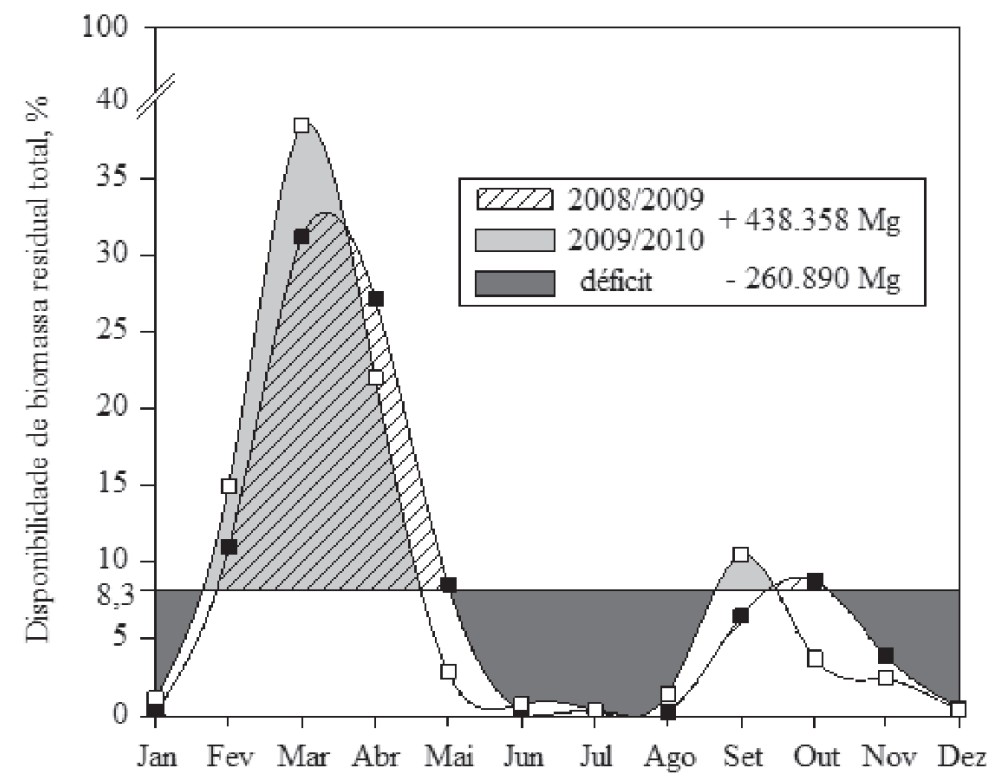

Figura 1: Excedente e déficit de oferta de biomassa residual das colheitas de milho, soja, trigo, cevada, aveia branca e aveia preta (\%), com potencial para produção energética, na região dos Campos Gerais do Paraná, nas safras 2008/2009 e 2009/2010. 

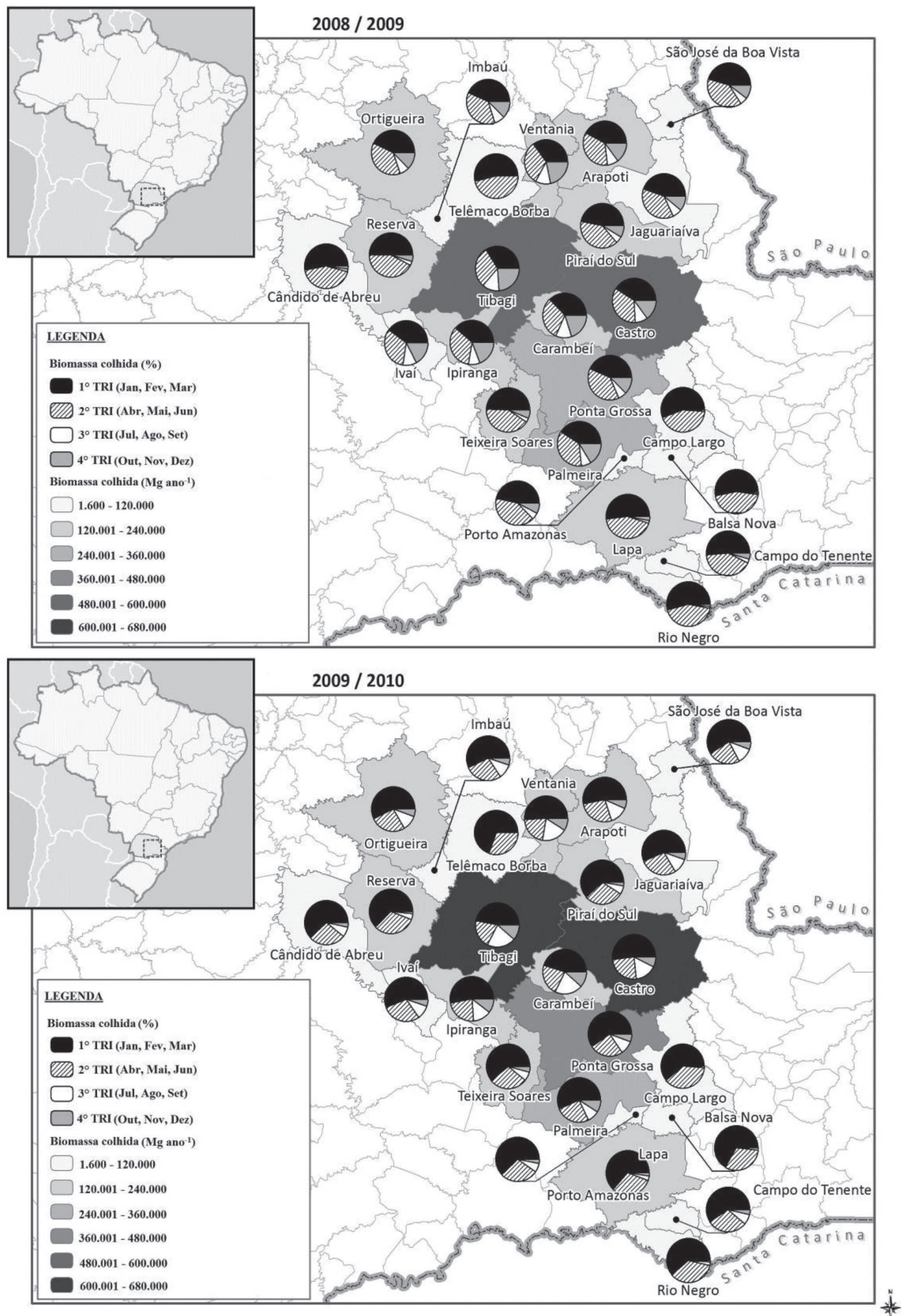

Figura 2: Disponibilidade média trimestral da biomassa residual agrícola, com potencial para produção energética, cultivada nos 24 municípios da região dos Campos Gerais do Paraná, nas safras 2008/2009 e 2009/2010. 
Tabela 4: Poder calorífico superior (PCS) e potencial teórico de produção de energia elétrica a partir da biomassa residual da colheita de grãos na região dos Campos Gerais, PR, Brasil, nas safras 2008/2009 e 2009/2010

\begin{tabular}{lcccc}
\hline \multirow{2}{*}{ Espécie } & PCS & & \multicolumn{2}{c}{ Potencial de produção de energia $(\mathbf{G W h})$} \\
\cline { 2 - 2 } & $\left(\mathbf{M J ~ K g}^{-1}\right)$ & & $\mathbf{2 0 0 8 / 2 0 0 9}$ & $\mathbf{2 0 0 9 / 2 0 1 0}$ \\
\hline Milho & 18,6 & $1.786,21$ & $1.796,22$ \\
Soja & 18,4 & $1.376,85$ & $1.630,26$ \\
Trigo & 17,3 & 433,27 & 418,60 \\
Cevada & 17,3 & & 28,15 & 35,12 \\
Aveia Branca & 18,1 & 50,53 & 64,31 \\
Aveia Preta & 18,1 & 257,77 & 240,62 \\
\hline Total & - & $3.932,77$ & $4.185,12$ \\
\hline
\end{tabular}

Matsumura et al. (2005) estimaram que a utilização dos resíduos da produção de arroz poderiam suprir aproximadamente $0,5 \%$ da demanda por eletricidade do Japão. Contudo, o panorama mais favorável observado nos Campos Gerais é reflexo das condições propícias ao cultivo agrícola, favorecendo a região frente aos cenários nacional e mundial quanto à exploração energética de biomassa da colheita de grãos, sem afetar a qualidade do manejo da cobertura do solo e a manutenção do plantio direto.

\section{CONCLUSÕES}

Os Campos Gerais do Paraná produziram 3.862.130 (2008/2009) e 4.107.932 Mg ha"1 (2009/2010) de biomassa residual total, com o cultivo das seis culturas analisadas. A maior produção de biomassa ocorre nos meses de março e abril, enquanto nos meses de junho a agosto a produção é insignificante.

A biomassa residual da colheita de grãos apresentou PCS comparável ao de espécies florestais, com potencial teórico total de produção de energia de 3.932 (2008/2009) e 4.185 GWh ano"1 (2009/2010).

Os municípios de Tibagi e Castro são os mais promissores da região dos Campos Gerais do Paraná para a instalação de termelétricas movidas a biomassa, por apresentarem maior produção e melhor distribuição sazonal dos resíduos.

\section{REFERÊNCIAS}

Andrews SS (2006) Crop residue removal for biomass energy production: effects on soils and recommendations. Disponível em: <http://soils.usda.gov/sqi/management/files/AgForum_ Residue_White_Paper.pdf $>$. Acessado em: 20 de novembro de 2013.

ABNT - Associação Brasileira de Normas Técnicas (1984) NBR 8633: Carvão vegetal: determinação do poder calorífico. Rio de Janeiro, ABNT. 13p.

Briedis C, Sá JCM, De-Carli RS, Antunes EAP, Simon L, Romko ML, Elias LS \& Ferreira AO (2012) Particulate soil organic carbon and stratification ratio increases in response to crop residue decomposition under no-till. Revista Brasileira de Ciência do Solo, 36:1483-1490.
CONAB - Companhia Nacional de Abastecimento (2013) Séries históricas de área plantada, produtividade e produção. Disponível em: <http://www.conab.gov.br/conteudos.php?a=1252\& ordem=produto\&Pagina_objemsconteudos=1\#A_objcmsconteudos $>$. Acessado em: 20 de novembro de 2013.

Carroll JP \& Finnan J (2012) Physical and chemical properties of pellets from energy crops and cereal straws. Biosystems Engineering, 112:151-159.

Correll D, Suzuki Y \& Martens BJ (2014) Logistical supply chain design for bioeconomy applications. Biomass and Bioenergy, 66:60-69.

Cuiping L, Yanyongjie, Chuangzhi W \& Haitao H (2004) Study on the distribution and quantity of biomass residues resource in China. Biomass and Bioenergy, 27:111-117.

Dodiæ SN, Zekiæ VN, Rodiæ VO, Tica NL, Dodiæ JM \& Popov SD (2011) Analysis of energetic exploitation of straw in Vojvodina. Renewable and Sustainable Energy Reviews, 15:1147-1151.

EPE - Empresa de Pesquisa Energética (2011) Balanço Energético Nacional 2011 - Ano base 2010: Resultados Preliminares. Rio de Janeiro, Ministério de Minas e Energia. 49p.

EPE - Empresa de Pesquisa Energética (2014) Balanço Energético Nacional 2014 - Relatório Síntese Ano base 2013. Disponível em : < https://ben.epe.gov.br/downloads/S\%C3\%ADntese\% 20do\%20Relat\%C3\%B3rio\%20Final_2014_Web.pdf>. Acessado em: 06 de agosto de 2014.

ESRI - Environmental Systems Research Institute, Inc. (2008) ArcGIS Geographic Information System Software. Editor 9.3. Redlands, CA.

Evans A, Strezov V \& Evans TJ (2010) Sustainability considerations for electricity generation from biomass. Renewable and Sustainable Energy Reviews, 14:1419-1427.

Fowler P, Krajaèiæ G, Lonèar D \& Duiæ N (2009) Modeling the energy potential of biomass - H2RES. International Journal of Hidrogen Energy, 34:7027-7040.

Höldrich A, Epp C \& Witzelsperger J (2009) Feasibility study for mixed biomass pellets production in the German sample region: Straubing, Bavaria. Munich, WIP Renewable Energies. 20p.

IBGE - Instituto Brasileiro de Geografia e Estatística (2011) IBGE@cidades, Lavoura Temporária 2009 e 2010. Disponível em: 〈http://www.ibge.gov.br/cidadesat/topwindow.htm?1〉. Acessado em: 05 de outubro de 2011.

Karaj S, Rehl T, Leis H \& Müller J (2010) Analysis of biomass residues potential for electrical energy generation in Albania. Renewable and Sustainable Energy Reviews, 14:493-499.

Kingwell R \& Abadi A (2014) Cereal straw for bioenergy production in an Australian region affected by climate change. Biomass and Bioenergy, 61:58-65. 
Kludze H, Deen, B, Weersink A, van Acker R, Janovicek K, De Laporte A \& McDonald I (2013) Estimating sustainable crop residue removal rates and costs based on soil organic matter dynamics and rotational complexity. Biomass and Bioenergy, 56:607-618.

Leal MRLV, Galdos MV, Scarpare FV, Seabra JEA, Walter A \& Oliveira COF (2013) Sugarcane straw availability, quality, recovery and energy use: A literature review. Biomass and Bioenergy, 53:11-19.

Lora ES \& Andrade RV (2009) Biomass as energy source in Brazil. Renewable and Sustainable Energy Reviews, 13:777-788.

Matsumura Y, Minowa $\mathrm{T}$ \& Yamamoto H (2005) Amount, availability, and potential use of rice straw (agricultural residue) biomass as an energy resource in Japan. Biomass and Bioenergy, 29:347-354

Melo MS, Guimarães GB, Ramos AF \& Prieto CC (2007) Relevo e Hidrografia dos Campos Gerais. In: Melo MS, Moro RS \& Guimarães GB (Eds.) Patrimônio Natural dos Campos Gerais do Paraná. Ponta Grossa, UEPG. p.49-58.

Nogueira LAH \& Lora EES (2003) Dendroenergia: fundamentos e aplicações. $2^{\text {a }}$ ed. Rio de Janeiro, Interciência. 199p.

Noro G, Scheffer-Basso SM, Fontaneli RS \& Andreatta E (2003) Gramíneas anuais de inverno para produção de forragem: avaliação preliminar de cultivares. Agrociência, 7:35-40.

Okello C, Pindozzi S, Faugno S \& Boccia L (2013) Bioenergy potential of agricultural and forest residues in Uganda. Biomass and Bioenergy, 56:515-525.

Parikka M (2004) Global biomass fuel resources. Biomass and Bioenergy, 27:613-620.

Powers SE, Ascough II JC, Nelson RG \& Larocque GR (2011) Modeling water and soil quality environmental impacts associated with bioenergy crop production and biomass removal in the Midwest USA. Ecological Modelling, 222:2430-2447.

Ruiz JA, Juárez MC, Morales MP, Muñoz P \& Mendívil MA (2013) Biomass logistics: Financial \& environmental costs. Case Study: 2MW electrical power plants. Biomass and Bioenergy, 56:260267.
Sá JCM, Santos JB \& Lal R (2012) An on-farm assessment of carbon monitoring and mapping scaling up in no-till fields. Disponível em: <http://www.fao.org/ag/ca/CA-Publications/ An_on_Farm_Assessment.pdf $>$. Acessado em: 01 de setembro de 2013

Scarlat N, Blujdea V \& Dallemand JF (2011) Assessment of the availability of agricultural and forest residues for bioenergy production in Romania. Biomass and Bioenergy, 35:1995-2005.

SEAB - Secretaria de Estado da Agricultura e do Abastecimento/ DERAL (2011a) Tabela Dinâmica de Produção Agrícola por Município. Disponível em: 〈www.seab.pr.gov.br〉. Acessado em: 07 de outubro de 2011.

SEAB - Secretaria de Estado da Agricultura e do Abastecimento/ DERAL (2011b) Estimativa de Safra. Disponível em: <www.seab.pr.gov.br>. Acessado em: 07 de outubro de 2011.

Shimandeiro A, Kantelhardt J \& Weirich Neto PH (2008) Characterization of major crop management in the buffer zone of Vila Velha State Park, state of Paraná, Brazil. Acta Scientiarum Agronomy, 30:225-230.

Silvestrin F (2011) Dinâmica de boro no solo e planta e sua influência na cultura do milho em dois latossolos de textura média. Dissertação de Mestrado. Universidade Federal do Paraná, Curitiba. 39p.

Tarkalson DD, Brown B, Kok H \& Bjorneberg DL (2009) Impact of removing straw from wheat and barley fields: a literature review. Better Crops, 93:17-19.

Telmo C \& Lousada J (2011) Heating values of wood pellets from different species. Biomass and Bioenergy, 35:2634-2639.

UEPG - Universidade Estadual de Ponta Grossa (2011) Dicionário Histórico e Geográfico dos Campos Gerais. Disponível em: <www.uepg.br/dicion/campos_gerais.htm>. Acessado em: 03 de outubro de 2011.

Valdez-Vazquez I, Acevedo-Benítez JA \& Hernández-Santiago C (2010) Distribution and potential of bioenergy Resources from agricultural activities in Mexico. Renewable and Sustainable Energy Reviews, 14:2147-2153. 